\title{
Tangence
}

\section{Les contextes de signification d'un fait divers}

\section{Jocelyn Létourneau}

Numéro 37, septembre 1992

Autopsie du fait divers

URI : https://id.erudit.org/iderudit/025724ar

DOI : https://doi.org/10.7202/025724ar

Aller au sommaire du numéro

Éditeur(s)

Tangence

ISSN

1189-4563 (imprimé)

1710-0305 (numérique)

Découvrir la revue

Citer cet article

Létourneau, J. (1992). Les contextes de signification d'un fait divers. Tangence, (37), 46-55. https://doi.org/10.7202/025724ar d'utilisation que vous pouvez consulter en ligne.

https://apropos.erudit.org/fr/usagers/politique-dutilisation/ 


\section{Lectures \\ d'un fait divers}




\section{Les contextes de signification d'un fait divers}

Jocelyn Létourneau *

La notion de fait divers est ambiguë: elle laisse supposer qu'un épisode rapporté n'a qu'une valeur secondaire, insignifiante et mineure alors que, en pratique, puisqu'il a été mentionné et mis en circulation dans l'espace public sous la forme d'une nouvelle digne d'intérêt, il est un artefact de la parole collective, un témoignage des regards et des discours que la société, par l'intermédiaire de ses hérauts médiatiques, porte et compose sur elle-même.

Un fait divers n'est pas * divers" pour au moins trois raisons. D'abord, si tel était le cas, il ne serait pas rapporté car on n'a que faire de monsieur L. qui, de manière routinière chaque matin après le petit déjeuner, change (déjà pour la seconde fois) la couche de bébé S. ${ }^{1}$. Par ailleurs, pour être relaté, un fait doit comporter en lui-même une certaine dose d'étrangeté, de singularité et de curiosité qui le rendra appréciable et reconnaissable comme phénomène ou agir insolite par le destinataire du message. Ainsi, un chien renversé par un automobiliste dans une rue de Québec n'entraînera pas de tergiversations et n'aura donc pas d'écho médiatique, à moins qu'un témoin ne s'empare de l'incident et ne le dramatise comme fait d'intérêt en le contextualisant par rapport à des topoï sociétaux; dans une autre société ou à une époque différente, la scène d'un chien mort pourrait revêtir, en elle-même, des significations qui décupleraient son intensité relative et la projetteraient au centre des débats. Enfin, toute matière de l'expérience humaine, animale ou extra-terrestre qui, au terme d'une décision éditoriale (chef de pupitre, responsable

- CÉLAT et Département d'histoire, Université Laval. L'auteur remercie son collègue Bohumil Jewsiewicki pour les remarques pertinentes qu'il a faites sur ce texte.

1 Bien qu'une telle action, banale pour une multitude de personnes vivant en Amérique du Nord au début des années 1990, pourrait acquérir le statut de fait divers s'il advenait que monsieur $L$. soit une vedette sportive ou de la scène, ou appartienne au clan des a gens riches et célèbres ", ou encore que monsieur L. soit, dans une société donnée, l'un des premiers hommes à s'acquitter de cette tâche. 
de l'information, etc.), acquiert le statut de fait divers, suppose un effort conscient de remodelage et de réactivation, sous une forme cohérente et racontable, d'actes ou de situations qui, autrement, se seraient normalement écoulés - et auraient été oubliés dans le flux ininterrompu des actions, des visions et des situations qui marquent la quotidienneté du genre humain; en d'autres termes, pour devenir fait divers - et nonobstant la banalité ou la singularité de l'événement survenu - quelque chose doit être construit comme tel et ce, à la suite d'une opération intelligente scandée par des phases de repérage, de sélection, de présentation, de contextualisation et de narration d'une matière brute ${ }^{2}$.

Si bien que l'on peut affirmer sans risque de se tromper qu'un fait divers est inséparable de son narrateur (situation d'énonciation) et de son destinataire (situation de réception), sa matérialité (sa facture et son contenu) obéissant à un contexte communicationnel, intertextuel et social d'ensemble - lui-même surdéterminé par la rumeur qui * infecte . l'espace dialogique de l'، émetteur * et du "récepteur * - qui conditionne et détermine la portée du fait divers, sa valeur et ses effets (son intensité médiatique).

Mais l'on peut dire beaucoup d'autres choses à propos du fait divers. Il est en effet pertinent de s'interroger sur les raisons qui poussent les organes de presse, transmetteurs de parole dans les sociétés massifiées, à relater ce genre d'information, voire à en faire le cœur de leur pratique éditoriale. À cet égard, notre hypothèse est que le fait divers est un aspect important de la régulation politicoidéologique à l'œuvre dans ces sociétés. En tant que mise en spectacle du quotidien, prétexte à une interrogation de la part des lecteurs (ou des auditeurs) sur leur vécu et discours raisonné de la société sur elle-même, le fait divers participe en effet de la formation du consensus au sein de ces sociétés et ce, de trois manières:

2 C'est pourquoi l'idée selon laquelle le fait divers est un cinclassable de l'information. n'a pas de pertinence. Le fait divers est, par définition, une matière extirpée du tohu-bohu de l'agir humain (ce qui suppose un choix conscient) et, en cela, une entité construite pour des fins précises (sensationnalisme, information générale, remplissage). Par ailleurs, un fait divers peut devenir, selon les changements qui surviennent dans l'espace médiatique formé par le contexte d'énonciation et de réception du message (rebondissements d'une nouvelle), et selon aussi le contexte communicationnel, intertextuel et social dans lequel il est situé, un événement majeur ou une nouvelle exceptionnelle, surtout s'il est envahi et porté par la rumeur publique. 
- en nourrissant la parole publique d'une matière qui amortit ou détourne, chez les participants d'un espace communicationnel, toute mobilisation pour la chose politique, la conséquence de cette situation étant d'accroître le * déficit démocratique * au sein des sociétés massifiées;

- en offrant une échappatoire à la condition aliénée d'une multitude de personnes par la transformation de l'excès, qu'il soit positif ou négatif, en un référent existentiel à envier ou à rejeter;

- en exerçant le jugement des personnes (lecteurs ou auditeurs) dans le sens d'un renforcement des positions conservatrices, de manière que la frontière marquant l'acceptable et le condamnable soit connue de tous (à défaut d'être acceptée).

C'est à partir de ces postulats que nous procéderons à la - lecture * d'un fait divers spectaculairement titré: *Une famille massacrée à la hache ", paru dans Le journal de Montréal du 20 février 1988, en essayant de faire ressortir comment cet épisode, réputé sans conséquence, est bien plus signifiant qu'il n'y paraît.

\section{Le fait divers comme mise en spectacle du quotidien}

La question qui nous occupe au départ est la suivante: pourquoi, d'un point de vue médiatique, avoir décidé de transformer cette information en nouvelle d'intérêt public, et pourquoi avoir choisi, parmi la pléthore d'informations qui sont quotidiennement mises en circulation par les agences de presse (ici l'Associated Press), celle-là en particulier pour la rapporter aux lecteurs d'un journal?

Une information est transformée en fait divers - et donc construite comme tel pour paraître dans un journal - si elle respecte différents critères. Elle doit d'abord s'inscrire dans la tradition d'information propre à ce journal ${ }^{3}$. Elle doit ensuite

3 Chaque journal se spécialise en effet dans le reportage de certaines nouvelles coïncidant avec les attentes de ses lecteurs. Chaque joumal établit par ailleurs une "barre sélective", sorte de critérium de tolérance éditoriale (on parlera ainsi de ajaunisme pour décrire une pratique journalistique ou une nouvelle relatée qui est en deçà des attentes éditoriales) par rapport à laquelle s'effectue le choix des informations rapportées. 
comporter une intensité médiatique exploitable ${ }^{4}$. Enfin, elle doit rejoindre la capacité présumée (ou antérieurement vérifiée) des lecteurs d'apprécier et de reconnaître, comme étant intéressante, une nouvelle 5 , et de l'intégrer à leur horizon communicationnel (donc de la réactiver dans l'espace public à travers leurs conversations quotidiennes).

C'est suivant ce contexte initial que doit être abordé notre fait divers. En soi, il est évident que l'épisode décrit — un enfant qui assassine quatre membres de sa famille 6 - comporte une singularité qui peut capter l'attention de lecteurs avides de sensationnalisme et habitués à en consommer en masse sous la forme d'images, de paroles et d'écrits. Si Le journal de Montréal n'est pas le seul quotidien à offrir en pâture à ses lecteurs des nouvelles sensationnelles?, il s'est néanmoins taillé une place à part dans un crêneau médiatico-informatif où le public visé consiste principalement en de larges segments des classes populaires, ouvrières et moyennes dont l'horizon communicationnel est fortement

4 Nous entendons par là la capacité d'une information à susciter en ellemême, étant donné son caractère singulier, un intérêt chez le lecteur et ce, nonobstant la capacité d'un rédacteur de nouvelles à créer ou à décupler cet intérêt par ses effets de présentation ou de style. En termes clairs, une information serait au départ dotée d'une valeur relative elle serait donc un capital à exploiter - qui se traduirait éventuellement par des bénéfices supplémentaires découlant de ventes accrues ou maintenues du journal.

5 C'est ce qui explique que toutes les informations mises en circulation dans l'espace privé des médias (les images téléscriptées) n'acquièrent pas le statut de fait divers (information portée, comme nouvelle, dans l'espace public). On peut en effet penser que, pour un journal à sensation ne rapportant que des actes d'agression, une bagarre dans un bar ne comportera pas, en elle-même, une intensité médiatique suffisante pour qu'elle soit décodée, interprétée et reconnue par le lecteur comme étant un fait divers digne d'intérêt. De même, habitué à soutenir une intensité médiatique extrême (une bagarre devant impliquer une violence inouie), ce lecteur ne reconnaîtra pas tout fait qui lui est présenté comme passant la barre de ses attentes.

6 Telle qu'elle est rapportée, la nouvelle ne permet pas au lecteur de savoir si la famille a été assassinée au complet puisqu'on laisse sousentendre, en prétendant que l'adolescent a tué l'un de ses frères et l'une de ses sceurs, que plus de trois enfants composaient la famille.

7 Le sensationnalisme est d'ailleurs la forme prédominante que prend la nouvelle dans les journaux en Occident et ce, peu importe la nature du journal. Que l'on traite de politique, de sport, d'arts ou de lettres, que l'on s'adresse aux élites ou au peuple, on recherche toujours l'exclusivité, le scoop, le choc. 
structuré par des faits relatifs aux sports, aux agissements excessifs (de personnalités mais aussi d'inconnus) et aux curiosités de la vie privée ou publique.

Le fait divers participe en réalité d'une vaste mise en spectacle du quotidien qui reflète le carnaval continuel de la condition humaine avec ses honneurs et ses déchéances, ses générosités et ses cupidités, ses joies et ses peines, ses passions et ses dénouements tragiques. Et, contrairement à ce que l'on croit (ou contrairement à ce que l'intelligentsia aime croire, façon d'autocélébrer son importance sociale et de créer son légendaire), c'est dans ce carnaval, véritable tableau kitsch du monde vécu, que se reconnait, s'identifie et se retrouve la masse des gens. On pourrait se perdre en conjectures sur les raisons expliquant l'engouement populaire pour le sport, pour les agissements exubérants et pour les curiosités de la sphère privée. Nous nous limiterons aux hypothèses suivantes: le sport est certainement l'un des forums principaux vers lequel sont dirigées, canalisées et absorbées les pulsions de masse. Depuis toujours lieu de rassemblement, si ce n'est support d'identité collective et de communion patriotique, le sport est aussi, pour de nombreux individus, un moment fort de leur agenda quotidien et une préoccupation vécue sur le mode personnel (sorte de transposition de soi dans le héros et vice versa). L'intérêt manifesté par nombre de lecteurs pour les agissements excessifs de personnages publics (dépenses somptuaires, paraître mondain, pratiques sexuelles inaccoutumées, cupidité, comportements agressifs, etc.) ou pour ceux de purs inconnus, traduit par ailleurs, vraisemblablement, une projection de leurs fantasmes ou de leurs exaspérations pour oublier leur condition aliénante. Enfin, l'attrait des gens pour les choses privées pourrait être interprété comme une sorte de voyeurisme dans lequel ils se commettent pour découvrir la condition scandaleuse, déprimante ou alarmante de l'autre, de manière à relativiser ou à réhabiliter leur propre sort.

Le fait divers ici analysé exprime et, tout à la fois, se situe au carrefour de ces conditionnements multiples qui déterminent la décision éditoriale de relater quelque chose qui est survenu. En tant qu'événement spectaculaire, il possède un potentiel médiatique exploitable et rencontre les exigences mercantiles recherchées. En tant qu'épisode dramatique de la vie d'un ménage, il permet à des lecteurs, badauds inassouvis, de pénétrer au cour de l'intimité d'une famille victime d'une tragédie majeure. Enfin, 
en tant que mise en scène du quotidien, il transforme inéluctablement une triste histoire en une nouvelle à sensation destinée à alimenter la "machine informative des sociétés massifiées, cette machine étant devenue, ni plus ni moins, l'agora où se déroule, nuit et jour, le spectacle tantôt pitoyable, tantôt réjouissant de la condition humaine.

\section{Le fait divers comme prétexte à une interrogation existentielle du lecteur sur son environnement familier}

Tablons sur ce premier plan d'analyse pour aller plus loin et montrer que le fait divers n'est pas qu'une banalité rapportée de l'expérience humaine. On a dit que l'un des critères fondant le fait divers tenait à la singularité de la situation décrite. Le meurtre de plusieurs personnes par un même assassin est certainement, en soi, un incident spectaculaire. Mais là ne s'arrête pas le drame: ce meurtre a été commis par un adolescent, dans une petite ville d'un État qui n'est pas réputé pour le nombre d'actes violents qui y sont commis ${ }^{8}$. Il s'agit en outre d'un État où la population blanche est fortement majoritaire, de souche germanophone en bonne partie, donc réputée disciplinée, laborieuse et rangée, et respectueuse de l'éthique protestante des rapports interpersonnels. Le plus étonnant, c'est que la tuerie s'est déroulée au sein d'une famille sans histoire, une famille aux mours tranquilles ${ }^{9}$, une famille modèle dont on se plaît à croire qu'elle est le cadre idéal pour l'éducation de citoyens responsables. Cette famille était par ailleurs heureuse - ne retrouve-t-on pas, sur la photographie accompagnant l'article, des visages épanouis, des sourires profondément francs, une complicité évidente entre papa, maman et les enfants? Bref, le tableau présenté n'a rien à voir avec les *cadres habituels * auxquels l'on associe d'ordinaire les déchaînements de violence gratuite et d'exaspération aveugle (aux États-Unis en tout

8 En 1988, le Minnesota venait au $40^{\circ}$ rang des États américains pour ce qui est du nombre de crimes violents (meurtres, viols, vols, assauts graves) par 100000 habitants commis sur son territoire, et au $44^{\circ}$ rang en ce qui a trait au nombre de meurtres (le District of Columbia étant ici considéré comme une unité administrative). Source: Statistical Abstract of United States, 1990, tableau n' 285, p. 171.

9 A cet égard, l'indice que toutes les personnes assassinées portaient des vêtements de nuit ne trompe pas, l'habillement étant ordinairement associé à la quiétude, à la sérénité et à la paix, et la nudité à l'obscénité, à la dégradation et à la tentation. 
cas): les milieux marginaux, les centres-villes, les communautés de noirs ou d'hispanophones, les familles monoparentales, etc. Au contraire, le crime a été perpétré par une personne apparemment saine d'esprit ${ }^{10}$, dans un environnement normalisé et sans qu'il y ait eu, de part et d'autre, provocations pouvant conduire à un geste aussi démesuré. N'est-ce pas là la condition de la famille type sous le règne de laquelle se range la famille québécoise? À vrai dire, dans cette histoire, tout porte à la confusion. On ne comprend plus. Pourquoi... ce massacre? Comment, dans un univers aussi commun, aussi conventionnel, archi-connu, un drame de cette envergure a-t-il pu se produire? Devant ces questions, le lecteur reste pensif et pantois. Dès lors, le fait divers n'est plus juste un incident sans intérêt. Il devient une énigme à résoudre (quels sont les motifs d'un acte aussi horrible?) et un prétexte pour s'interroger sur son propre vécu (pourquoi eux? c'aurait pu être nous! se pourrait-il que ce soit nous un jour?). Nous touchons là un second contexte conditionnant la relation du fait divers: parce qu'il interroge le lecteur sur ses propres certitudes (confiance en ses proches, confiance en son milieu), celui-ci n'est plus décodé comme un fait divers mais comme une éventualité plus ou moins plausible contre laquelle il faut se prémunir.

\section{Le fait divers comme discours raisonné de la socièté sur elle-même}

Si le fait divers interroge le lecteur dans ses certitudes, il le réconforte aussi dans ses inquiétudes concernant les excès de toutes sortes qui marquent et qui rongent la condition humaine, notamment en cette période de temps troubles où l'insécurité s'installe dans tous les pores du tissu social.

Tel qu'il est présenté et rapporté, le fait divers renvoie en effet à un certain nombre de thématiques sociétales qu'on discute largement dans l'espace public: celle, par exemple, de l'absurdité de la violence, celle de la révolte des jeunes contre l'autorité parentale, celle de l'incompréhension et de la tension à l'intérieur des familles, celle de la délinquance juvénile, etc. Par ailleurs, les détails qu'on utilise pour construire le fait divers actionnent le jugement populaire et le fixent sur des positions conservatrices, en ce sens que tout l'incident est décrit de manière que l'on

10 Rien ne permet de croire en effet qu'avant cet épisode dramatique, le garçon ait manifesté quelque déséquilibre sur le plan comportemental. 
réagisse négativement, *impulsé. par un profond écœurement, totalement déconcerté, sans possibilité de recourir au contrepoids de la pensée raisonnable. Cela montre à quel point le fait divers est un regard et une évaluation que la société porte sur ellemême et ce, suivant une subtile morale.

Dans la présentation qui nous est faite de la tuerie, de ses motifs possibles et de ses suites, on donne des détails particuliers. On nous dit par exemple que *le garçon s'était en partie rasé la tête et avait teint le reste de ses cheveux en noir avant que les cadavres ne soient découverts.. On rapporte également qu'sune hache ensanglantée a été découverte dans la cave de la maison. Troisièmement, on mentionne, en ayant recours au témoignage d'un policier (quoi de plus crédible que la parole d'un agent de la paix, surtout aux États-Unis ${ }^{11}$ ?), que le tueur avait des problèmes avec son père en raison d'une cassette *qu'il avait achetée et qu'il ne voulait pas que son père écoute. Enfin, on précise que le procureur du comté demandera que le garçon soit jugé comme un adulte.

Bien qu'il n'ait aucune chance de faire valider ses spéculations (il n'est pas sûr en effet que ce fait divers ait des suites dans l'édition suivante du journal), le lecteur peut monter tout un scénario pour répondre à la question qui l'étreint: pourquoi ces meurtres? Il peut se dire que le garçon adhérait sans doute à quelque idéal pernicieux ou qu'il était membre d'un groupe préconisant la violence comme forme instituée de ritualisation; ou que celui-ci, une fois son geste commis (peut-être pour attirer l'attention sur sa personne), avait décidé de lui donner un sens particulier en s'attribuant les marques identitaires distinctives des "néo-desperados ". À cet égard, l'indice du crâne rasé et celui des cheveux teints ne trompent pas. Le crâne rasé est un symbole de révolte ou de servilité: chez les militaires, il indique une obéissance aveugle à l'ordre imposé d'en haut; chez les rebelles, il exprime un jusqu'au-boutisme délirant. Quant à la teinture noire,

11 Il $\mathrm{y}$ aurait une hypothèse à fouiller selon laquelle, aux États-Unis, les figures habituelles de la Sainte-Trinité (le Père, le Fils et le Saint-Esprit) ont été remplacées par trois autres, à savoir le Policier, l'Ambulancier et le Pompier, dont les apparitions et les interventions soudaines (médiatisées à outrance - qu'on pense aux émissions telles Urgences, Surviure, Rescue 911 et bien d'autres encore) s'enflamment du cri strident des sirènes qui les annoncent, des phares gyroscopiques qui les illuminent, des rassemblements de curieux qu'elles provoquent et de l'excitation générale qu'elles entraînent. 
elle traduit la volonté de s'enduire l'esprit d'un goudron mortuaire qui rend compte d'un désir de raccordement avec les forces du mal. À moins que ce grotesque maquillage n'ait été finalement qu'un naîf camouflage pour échapper à la poursuite policière...

La découverte d'une hache ensanglantée dans la cave de la maison montre, de son côté, le caractère absolument horrifiant, sadique et barbare du geste. Non seulement a-t-on affaire à un garçon qui commet le meurtre suprême de tuer ses parents (qui sont ses géniteurs autant que ses tuteurs), mais il le fait en outre d'une manière primitive, dans un geste où le contact direct avec les victimes, les éclaboussures provoquées par le sang qui gicle de partout, leurs cris désespérés au moment où ils se sentent mourir dans la souffrance des coups répétés, rappelle les tortures les plus sordides de l'histoire. Or, le bourreau est le fils, l'héritier, le porteur du patrimoine familial, le transmetteur de la mémoire du groupe. Dans un geste insensé, contre nature, celui-ci met fin à tout, empêche l'avenir d'éclore, éteint toute possibilité de continuité. La famille est détruite. La structure initiale de sociabilité dans le monde moderne est effacée. C'est le vide et le chaos.

Devant ce nihilisme déroutant, il importe de se raccrocher à un espoir, c'est-à-dire à une cause, pour expliquer le drame. Il doit y avoir une raison derrière toute cette violence gratuite. Peu importe que le geste soit condamnable, inacceptable, anormal, il a dû être conçu comme une solution à une terrible impasse. Le contexte est confus: on parle d'une cassette achetée par le fils et dont le contenu était inacceptable pour le père. Faible prétexte pour tuer, a fortion pour décimer toute une famille dont plusieurs membres étaient sans doute ignorants du conflit déclaré ou appréhendé entre le fils et le père. Cela dit, la cause a beau être existante, elle n'en demeure pas moins insignifiante par rapport à la démesure du geste: le meurtre de quatre personnes innocentes est un acte impardonnable.

D'où le jugement lapidaire: bien que commis par un adolescent, les meurtres sont d'une telle gravité par leurs incidences qu'ils doivent être punis de manière extrêmement sévère, exemplaire. Cette information est rassurante. À ce moment du reportage, le lecteur se réconcilie avec sa conscience. Il retrouve les limites du bon sens, là où il se sent à l'aise, là où il puise sa force pour rester, en dépit des excès secrétés par un monde tourmenté, un être civilisé et normal. La transgression des lieux communs ne peut rester impunie. Elle réclame un châtiment décisif. 
On voit bien, en suivant ce parcours narratif scandé par un fait relaté, sa cause appréhendée, le contexte de son déroulement et ses suites possibles, comment le fait divers, nonobstant la réalité (d'ailleurs incontrôlable) à laquelle il renvoie, est construit suivant un certain nombre de paramètres qui font que le lecteur peut se retrouver dans une structure de compréhension et de mise en relation des choses qu'il maîtrise et par rapport à laquelle il peut se situer.

\section{Conclusion}

On a vu dans les pages précédentes à quel point, si on le soumettait à un *interrogatoire ", le fait divers était un révélateur de la société discutant d'elle-même, s'exposant et se jugeant. Contrairement à l'impression première, le fait divers n'a rien d'un épisode banal et sans conséquence dont on rend compte pour - remplir " un journal... si ce n'est des lecteurs. Il est davantage un texte repère, un texte référence, en ce sens qu'il établit les limites entre le bien et le mal, entre l'acceptable et le condamnable. Le fait divers provoque le jugement du lecteur, exerce sa vigilance et le rassure sur ses propres choix et sa propre façon d'être. On pourrait penser que le fait divers est un rempart de la société contre les excès qu'elle sécrète. C'est dire son importance. C'est savoir surtout à quel point il faut être attentif à ses messages. 